\title{
Recent Areas of Academic Research in Environmentally Conscious Manufacturing and Future Trends
}

\author{
Surendra M. Gupta \\ Northeastern University, USA \\ s.gupta@northeastern.edu
}

\begin{abstract}
We all would like to maintain the current and future environmental protection, economic profitability and quality of living standards. This has created a buzzfor Environmentally Conscious Manufacturing and led to a goal for sustainability which is the ability to continue the current behavior indefinitely. This has led to a push for reduction and elimination of waste as well as preservation of resources which is the mission of product recovery using reverse supply chains. Reverse supply chains consist of a series of activities required to collect used products from consumers and reprocess them to either recover their leftover market values or dispose of them. Remanufacturing is an important element of reverse supply chains. The increase in the popularity and implementation of remanufacturing have created many challenges due to uncertainties in the quality and quantities of returned products, difficulties in estimating the remaining lives of the components, unknown timings of the availability of end-of-life products, balancing the remanufacturing line, pricing decisions, warranty cost estimation and opportunity for committing fraud by third party and customers, to name a few. This talk will provide an overview of the various modeling techniques used by researchers to address the above-mentioned challenges as well as avenues for future research.
\end{abstract}

